\title{
Latifundio y territorio: reflexiones en torno a la reforma agraria en Colchagua, 1960-1973.
}

\author{
Jorge Olea Peñaloza \\ Universidad Católica de Chile,Santiago, Chile \\ Email: jmolea@uc.cl
}

\begin{abstract}
Resumen: La Reforma Agraria se ha entendido como un proceso de transformación productiva, destacándose desde la historiografía como el punto de inflexión en la desaparición del latifundio. Sin embargo, al reflexionar el proceso desde el concepto de territorio resalta la necesidad de afrontar su interpretación desde otra perspectiva. Para ello, se propone estudiar la Reforma Agraria como un conflicto producto de una apropiación hegemónica histórica de los recursos naturales, proponiendo una distinción entre hacienda y latifundio. Asimismo, se propone discutir sobre la actualidad del latifundio, pues el proceso de Reforma Agraria habría sido solo el fin de la hacienda más no el de la gran propiedad.
\end{abstract}

Palabras claves: Reforma Agraria, territorio, agroecosistemas, Colchagua

\section{Large estate and territory: reflections about the agrarian reform in Colchagua, 1960-1973.}

\begin{abstract}
Agrarian Reform has been understood as a process of productive transformation, standing out from historiography as the turning point in the disappearance of the large estates (latifundio). However, reflecting about this process from the concept of territory underlines the need to confront its interpretation from another perspective. To do so, the approach is to study the Agrarian Reform as a conflict coming from a historical hegemonic appropriation of natural resources, proposing a distinction between hacienda and latifundio. Likewise, it intends to discuss the current situation of the latifundio, since the process of Agrarian Reform has only been the end of the hacienda and not the end of large property.
\end{abstract}

Keywords: Agrarian reform, territory, agroecosystem, Colchagua

\section{Latifúndio e territórios: reflexões sobre a reforma agrária em Colchagua, 1960-1973.}

Resumo: A reforma agrária tem sido entendida como um processo de transformação produtiva, destacando-se a partir dos historiadores como o ponto de viragem no desaparecimento de grandes fazendas. No entanto, refletindo o processo a partir do conceito de território destaca a necessidade de abordar a sua interpretação de uma outra perspectiva. Para este fim, propomos estudar a reforma agrária como um produto de um conflito apropriação histórico hegemônico dos recursos naturais, propondo uma distinção entre riqueza e grandes propriedades. Propõe-se também a discutir hoje os latifúndios, já que o processo de reforma agrária teria sido apenas 
para a fazenda, mas não a grande propriedade

Palabras-chave: Reforma Agrária, territorio, agro-ecosistema, Colchagua

$* * *$

\section{Introducción}

El siguiente trabajo parte de dos preguntas muy sencillas ¿qué es el latifundio y cómo es su funcionamiento? Durante la discusión de la Reforma Agraria en Chile el objetivo central era terminar con la gran propiedad y todo el régimen de dominación que éste poseía, y para ello era fundamental el poder redistribuir las tierras que estaban concentradas en pocas manos, y que a su vez, representaban una explotación deficiente desde el punto de vista agropecuario. De esta manera, se buscaba acabar con los "predios mal explotados”, es decir, "aquel cuya explotación se realiza en condiciones económicas, técnicas o sociales inadecuadas” y con los “predios abandonados”: “aquel que no es objeto de hechos positivos de explotación económica como cultivos agrícolas, empastadas, crianza de ganado, cuidado y conservación de los bosques naturales o artificiales, u otros de análoga significación económica” (Ley 16.640 de Reforma Agraria, 1967).

Con la Reforma Agraria se acabó con casi cuatrocientos años de una estructura productiva y social que había hegemonizado una importante porción del territorio chileno (Bengoa, 2015). Asimismo, en un corto plazo, la Reforma Agrariasignificó una profunda restructuración en las formas de tenencia de la tierra y en los sistemas productivos asociados a la actividad silvoagropecuaria. En otras palabras, se logró la reorientación productiva de los predios agrícolas y de la ruptura definitiva de la sujeción de parte del campesinado a quienes detentaba la propiedad de la tierra.

Sin embargo, esa transformación impulsada por la Reforma Agraria se vio interrumpida abruptamente tras el golpe de Estado ocurrido en septiembre de 1973, el cual impulsó un nuevo proceso reformador en el espacio rural. Este proceso, no obstante, estuvo marcado por nuevos matices respecto a las formas en que se llevaría a cabo el mejoramiento del campo, cuyo centro ya no estaría en la redistribución de la tierra, sino en la modernización de los modos de producción, así como en la intensificación del uso de recursos y capital (S. Gómez \& Echeñique, 1991).

\section{Genealogía del valle central: latifundio, hacienda e historiografía}

La idea central de la Reforma Agraria chilena era modificar, sustancialmente los derechos sobre la tierra que venían desde la época colonial, ya que tal como lo demostró Bengoa en su estudio sobre las casas de la Hacienda Quilpué, es posible rastrear la continuidad de la tenencia de 
la tierra hasta el período de la conquista(Bengoa, 1990). Es esta condición de estabilidad la que ha dado la connotación de la gran propiedad como parte sustantiva de la identidad nacional. La literatura, por ejemplo, ha denominado al latifundio como la "Casa Grande", donde Luis Orrego Luco (1908) transmuta la imagen de una casa patronal y su historia con la de Chile, con la idea de que el país no sería más que una gran hacienda. Por su parte, José Donoso (1978), hará transcurrir en su "Casa de campo" la misma idea, pero ya más decadente y en vías de desaparecer, donde la hegemonía de la gran propiedad solo se sigue sosteniendo en función de dispositivos culturales, de una inercia sin escapatoria, aceptada tanto por los que se beneficiaban como por los que se veían profundamente perjudicados.

Estos significativos ejemplos nos dan cuenta de un fenómeno mayor, y que ha sustentado de alguna manera el imaginario chileno. Por una parte, la idea de la total hegemonía del latifundio en el transcurso de la historia de Chile, incluso más que como sistema productivo, sino que como articulador del orden social. La mayoría de los estudios concentrados en la agricultura en Chile están más vinculados a aspectos sociales y de discusión sobre lo que implica la organización de la sociedad rural en relación a la hacienda. En ese sentido, resulta clave la obra de Mario Góngora quien de alguna forma sintetiza esos postulados en sus tres obras principales, la cual parte en la búsqueda de los orígenes de los inquilinos (Góngora, 1974), para luego discutir sobre la movilidad de los sujetos sociales en lo que denomina la "sociedad fronteriza" (Góngora, 1966) y, finalmente, enfocarse en el origen de la gran propiedad (Góngora, 1970). En esta trilogía de estudios sobre la conformación de la sociedad rural, el autor logra dar forma a la idea de cómo la actividad hacendal configura la totalidad de las relaciones sociales y productivas de la zona central de Chile.

Por otra parte, laidea de la supremacía del paisaje agrario asociado a la hacienda, como imagen representativa de la ruralidad chilena, bajo la prerrogativa de que sería el denominado Valle Central, el modelo de paisaje sobre el que se edificaría el país, pues tal como se necesita de un mito fundacional para dar curso a la narración de la nación(Bhabha, 2010)también sería necesario tener un paisaje mítico, que permita dar una coherencia a la narración, y que se transforme en el modelo hacia el cual debiesen transformarse los demás paisajes.Sobre la construcción de este imaginario en función del paisaje, se ha estudiado desde un punto de vista literario (Schoennenbeck, 2013) y concentrado en la creación de ciertos imaginarios, pero la hipótesis respecto a la expansión de esa idea de paisaje al resto de los territorios aún queda por desarrollarse.

Es por esto que sería necesario indagar respecto a una genealogía de la idea de este latifundio para poder aportar algunos elementos a la discusión del valle central, y avanzar en el objetivo de identificar las especificidades del latifundio como núcleo histórico y geográfico de lo que se esperaba derribar con la puesta en marcha de la Reforma Agraria. 
El latifundio ha sido esgrimido como representante, casi único, respecto al atraso económico y social de Chile: "Nada de ello (incorporación de nuevas técnicas y desarrollo del mercado), sin embargo, sirvió para modificar la base misma de la producción agraria: ni su escasa tecnificación interna, ni su tendencia a la concentración de la propiedad, ni su dependencia de una mano de obra semi-servil” (Pinto \& Salazar, 2002, p.106). El problema del latifundio, no sería un tema de incentivos clásicos, como el aumento de productividad o énfasis en la inversión tecnológica, sino que estaría sustentado en criterios de otro orden: "No sería entonces, de acuerdo a esta argumentación, la falta de incentivos económicos la que inhibió al régimen hacendal de incrementar y modernizar su producción, sino consideraciones de orden más político y estructural” (Pinto \& Salazar, 2002, p. 111).

La primera obra que describió sistemáticamente la situación de la agricultura en Chile es la elaborada por Claudio Gay (1862) a mediados del siglo XIX. En ella, se muestra una detallada descripción del mundo rural, tanto de su ámbito social como productivo, concentrado su análisis en la gran propiedad y relevando principalmente dos cuestiones: las características y condiciones de los trabajadores rurales, destacando las diferentes funciones que existían al interior de las haciendas; y por otro lado, al atraso técnico, el que llamó profundamente su atención, pues estaba relacionado tanto con la baja productividad del sector agrario, pero también con las pésimas condiciones en que se encontraban sus trabajadores.

En el año 1938 sale publicado el texto de George McBride que será la referencia obligada para cualquier debate o análisis del sector agrario durante el siglo XX. Con el título “Chile: su tierra y su gente” se presenta un desolador panorama de la realidad chilena, cuyo énfasis principal está en las malas condiciones de los habitantes rurales y la desidia de los grandes propietarios, tanto con sus trabajadores como con sus campos. En este libro, McBride ubica el origen del campesinado a fines del período colonial, con la consolidación de una estructura social basada en la hacienda, cuando: "los propietarios habían convertido en inquilinos a los esclavos, a los araucanos cautivos y a los indios encomendados”(McBride, 1973, p. 114). El origen social de este sector estaba acotado a los estamentos subyugados de la época colonial, por lo que representaría una manifestación -ahora republicana- de un orden que siempre ha imperado. Será la primera vez que alguien plantea que: "La hacienda chilena llegó a su completo desarrollo y sobre ella se organizó la vida entera del país”(McBride, 1973, p. 96).

Esta lectura se ve reforzada por diversos analistas sobre el tema de la agricultura y su desarrollo a lo largo del siglo XX. Para Ballesteros (1965) luego de la gran depresión de los años treinta, existió la posibilidad de mejorar las condiciones en que se venía dando la agricultura, para lo cual el Estado tomó una serie de medidas, las que sin embargo, tuvieron una implementación deficiente, sobre todo de las innovaciones técnicas que eran necesarias. Asimismo, Santana (2006) analiza al sector agrario chileno como una crisis agrícola permanente en la medida que no logra consolidar ciclos de acumulación tecnológica ni productivos. Bauer (1994) reforzará 
esa idea, al plantear que el sector agrícola presenta como característica fundamental el desarrollarse bajo una modernización parcial, en donde solo algunos sectores logran los avances técnicos necesarios, pero sin alcanzar a parte significativa del mundo agrario. Este argumento es replicado por Bengoa (2015) quien habla de una modernización incompleta, bajo los mismos criterios que Bauer. Para Millar y Fernández (2008), este panorama tendría como causa la fuerte presencia estatal en los mercados y en la producción, así como en la falta de incentivos y pérdida constante de competitividad respecto a los demás sectores de la economía.

\section{Territorialidad del latifundio}

¿Cómo abordar un análisis del latifundio? La propuesta en esta investigación fue considerarlo desde una perspectiva territorial. Para ello, se siguen tres ejes: una economía política de los territorios;es decir, un análisis de las relaciones sociales de la producción que rigen el modo en que se desarrollan y confrontan las clases representantes del trabajo y del capital, lo cual involucra de manera directa al espacio(Raffestin, 2013). En segundo lugar, la territorialidad, en cuanto condición ineludible para el surgimiento del territorio, pues es necesario que el espacio se territorialice a través de la apropiación y que en él se manifiesten las múltiples fuerzas en disputa por el territorio(Haesbaert, 2013). Por último, la apropiación de la naturaleza, ya que en cuanto espacio agrario, los mecanismos de valorización y transferencias de valores funcionarían de manera distinta a las que presenta el resto de la materialidad que circula en la formación y reproducción del capital, donde los recursos naturales son parte central de dicho sistema productivo(Foster, 2004).

A partir de esto, la discusión gira en torno a la producción del espacio agrario, que consiste en un giro en la perspectiva analítica de la economía política de la producción. Desde un punto de vista marxista, la economía política se puede entender como el análisis de las relaciones sociales de producción, que rigen el modo en que se desarrollan y confrontan las clases representantes del trabajo y del capital, lo cual involucra de manera directa al espacio (Lefebvre, 1978). Según Lefebvre, esto ha funcionado en la medida que dichas estructuras en conflicto permanecen sólidas, pero que resulta limitado en momentos de crisis. Dicho de otro modo, cuando se pasa de la producción en el espacio a la producción del espacio es necesario prestar atención al desdoblamiento del concepto de la producción, tanto la producción de mercancías como la producción de ideas (Lefebvre, 2013).

Siguiendo a esta perspectiva, y para analizar el territorio como espacio producido se puede recurrir a Raffestin quien plantea que para que el territorio aparezca es necesario que el espacio se territorialice, a través de la apropiación, y que en él se manifiesten los múltiples poderes (Raffestin, 2013). De este modo, se busca entender al territorio como algo que se construye en base a relaciones sociales, el cual se encontraría determinado en distintos grados por las estructuras materiales y los acontecimientos, pero 
además por sentidos e información. En síntesis, la propuesta de Raffestin es que cuando se produce una apropiación de un espacio -ya sea concreta o simbólica- estamos en presencia de una territorialización. A partir de allí, vendrán los procesos de transmutación territorial y de los “múltiples poderes” que actúan en un espacio.

Asimismo, y siguiendo a Santos (2000), los procesos de construcción y desarme de territorios no son definitivos, sino que hay que mirar más profundamente cada elemento analizado, y aún más, con una perspectiva histórica mayor, poniendo mayor atención a los procesos de adscripción y desarrollo técnico. En esta línea, se rescata la propuesta de las "prótesis" espaciales: "La configuración territorial está formada por el conjunto de sistemas de ingeniería que el hombre va sobreponiendo a la naturaleza, verdaderas prótesis, de manera que permita la creación de condiciones de trabajo propias a cada época. El desarrollo de la configuración territorial en la fase actual viene con el desarrollo exponencial del sistema de transportes y del sistema de telecomunicaciones y de la producción de energía” (Santos, 2000, p. 41).

El concepto teórico que nos permite aproximarnos a este problema es el de territorio. Si bien se reconoce que el concepto ha ido asimilándose cada vez más a la idea de espacio geográfico de tipo más genérico, se busca rescatar el debate teórico que lo contiene y aplicar dicha discusión al análisis de las transformaciones acaecidas en el mundo agrario vinculado al espacio agrario(Altschuler, 2013). Para Benedetti (2011) el territorio ha seguido dos grandes líneas, la naturalista que rescatando la visión más cercana a Ratzel (1982)plantea el estrecho vínculo entre el territorio y una comunidad, y cómo ésta construye una organización para administrarla; y por otro lado, un acercamiento geográfico al territorio de corte geopolítico, que va generando dispositivos de planificación y administración, en base a áreas delimitadas por la administración estatal. La potencialidad analítica del territorio tiene como fortaleza además, la posibilidad de indagar en distintas escalas, lugares o redes, pudiendo considerarse de manera conjunta y relacional a todas esas dimensiones, para lograr una perspectiva mayor de las relaciones socioespaciales en el marco que permita entender las dinámicas que impone el capitalismo contemporáneo, como aparato político, económico y social (Jessop, Brenner, \& Jones, 2008).

\section{Territorios agrarios}

La trayectoria de un territorio es posible de investigar desde distintas perspectivas. Para Alan Baker existe un vínculo entre geografía e historia que es necesario de desentrañar y que opera en cada uno de los territorios de manera distinta. Existiría una dualidad entre elementos que se podrían considerar comunes, "locaciones de las geografías y las historias” y, al mismo tiempo, tres enfoques que deben adaptarse según el caso que se esté estudiando: las geografías del ambiente, del paisaje y de la región (Baker, 2003). Esto implica realizar una lectura crítica de los espacios y de sus funcionamientos, adecuando las preguntas no tan sólo a los elementos 
que se buscan relevar por parte del investigador, sino que profundizando en los aspectos del propio territorio.

Por otra parte, existe una mirada más casuística, donde el enfoque está puesto en las particularidades de cada territorio. En ese sentido, es posible buscar la transformación de los territorios en sí, de manera morfológica y de sus funcionalidades. Esa línea iniciada por Carl Sauer ${ }^{1}$ (1940), buscaba comenzar por los aspectos físicos del espacio geográfico para luego articular el uso social de ese espacio. Una de las obras fundamentales es la Identidad de Francia del historiador Fernand Barudel, quien dedica su primer tomo -de tres en total- a relevar los aspectos físicos de aquel país, y presentarlos como uno de los antecedentes básicos en la conformación de lo que se podría denominar “lo francés” (Braudel, 1993). A partir de lo físico se comienza un proceso de habitabilidad del espacio, el cual a su vez irá forjando la ocupación de los otros espacios, de manera acumulativa, a lo largo de la historia (Urteaga, 1998). Esto puede entenderse a través de los cambios en la configuración de los paisajes, principalmente al considerarla como la parte visible o perceptible de los usos del espacio, siendo la parte material uno de sus principales estructuras que le dan forma (Garrabou \& Naredo, 2008).

Un espacio rural está compuesto por la interacción entre un sistema natural y un sistema social, donde el objetivo principal es la obtención de recursos naturales para la reproducción humana. Sin embargo, esa relación está supeditada a las condiciones que el medio ofrece, pues las actividades rurales dependen de factores que no están en control humano, como las precipitaciones caídas, las condiciones naturales del suelo, la cantidad de irradiación solar, entre otros factores. Es por esto que se plantea que la manera más precisa para referirse a estos sistemas es el concepto de agroecosistema, donde el ecosistema natural está siendo modificado por el ser humano, en interrelación a los factores externos a la actividad humana que siguen influyendo en su desarrollo. Es en ese sentido, el análisis de los agroecosistemas, se enfoca en la interacción entre el sistema social y el natural, y a su vez, representa la magnitud e intensidad con la que se transforma el medio para fines humanos.

\section{Reforma agraria y territorios agrarios}

En el caso de la Reforma Agraria se trata de modificar, a partir de una reorganización de los sistemas de tenencia, el sistema social. Sin embargo, resulta necesariopreguntarse por el funcionamiento productivo de lo que se reformó, y al mismo tiempo, cuáles son las estructuras sociales y naturales que estaban relacionadas con esos sistemas de explotación. Si seguimos la argumentación de que los espacios son producidos en su relación con la acumulación de capital, la hacienda como sistema productivo respondía a un tipo de acumulación y de apropiación de capital, que en base a una explotación rentista se mantuvo durante mucho tiempo. Por lo tanto, intervenir ese tipo de organización espacial tendría consecuencias no tan solo en la organización social -que era uno de sus objetivos principales- 
sino que también se vería afectado la organización de la explotación de los recursos naturales. En otras palabras, la restructuración productiva que se buscaba con la Reforma Agraria, tuvo repercusiones en la intensidad de la exacción de recursos desde y a partir de la tierra.

Para ello es indispensable partir desde la base productiva de la hacienda (Figura $\mathrm{N}^{\circ} 1$ ). El sistema hacendal se componía de una agricultura de base orgánica, donde la mayoría de sus flujos energéticos y materiales estaban al interior de su sistema(Campos \& Naredo, 1980). Las plantas cultivadas y los animales criados en la hacienda representaban la mercancía que circulaba en el mercado, pero al mismo tiempo, éstas eran el punto de partida de otros procesos, como la alimentación, la descomposición, la fuerza motriz o calórica. Desde fuera venían principalmente algunos abonos sintéticos y fuerza motriz mecanizada (Garavaglia, 1989; González de Molina \& Toledo, 2014; Naredo, 2001).

\section{Figura $\mathrm{N}^{\circ} 1$ \\ Sistema productivo hacendal interno}

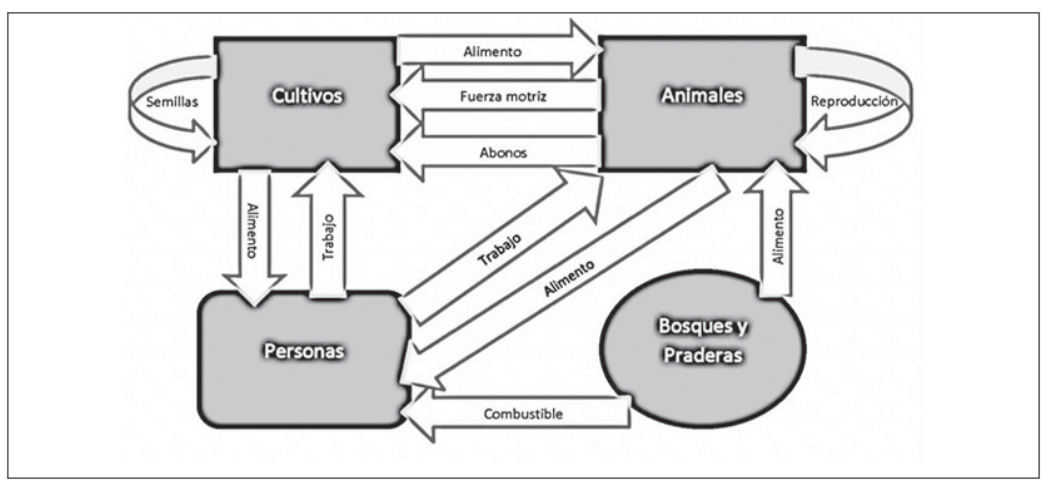

Fuente: Elaboración propia

Además, la explotación agrícola de la hacienda estaba en directa relación con el tipo de cultivo que se quería desarrollar, las aptitudes agrológicas de cada lugar y, por último, de la disponibilidad de tierras. El método que se desarrollaba en la hacienda correspondía a la rotación, el cual consistía en ir alternando tipos de cultivos y usos de una porción de terreno, articulándose con los demás sectores de la hacienda. Un ejemplo de ello lo podemos ver en la Figura $\mathrm{N}^{\circ} 2$, en el que un mismo potrero es utilizado de distinta forma año a año, en un período de cinco años en total. La hacienda por lo tanto necesitaba una superficie mayor para poder desarrollar este sistema productivo, pues la circulación interna de energías y materiales necesitaban tasas de descanso de tierras, o de renovación de aptitudes agrológicas, mucho mayores que en sistemas más intensivos. 


\section{Figura $\mathrm{N}^{\circ} 2$ \\ El sistema de rotación}

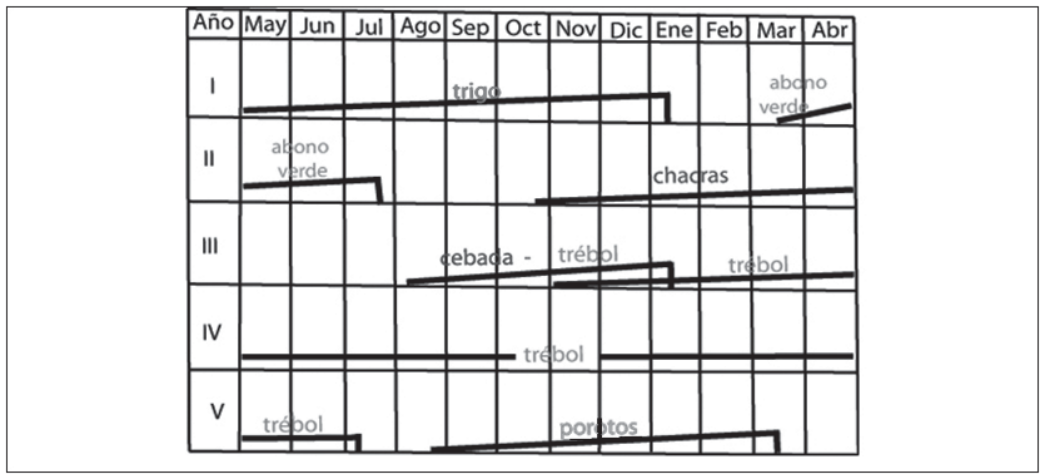

Fuente: Reproducción del autor a partir de: Carreño, Alfonso (1940) Monografía cultural y económica de la hacienda Santa Mariana, Rengo, Colchagua

En síntesis, la historia de un sistema agrícola como la hacienda debe examinar, en primer lugar, los procesos asociados a los cultivos; su período de siembra y cosecha; los sistemas tecnológicos asociados, el grado de injerencia del desarrollo de las técnicas de cultivo y sus características; los sistemas sociales, económicos, culturales asociados a esas técnicas, las formas de organización de las comunidades, las relaciones de poder, los sistemas laborales y las redes de sociabilidad. Todos estos elementos están actuando en el proceso de territorialidad y en es lo que está en conflicto con la aplicación de la Reforma Agraria.

\section{Colchagua y la Reforma Agraria}

Para Roberto Santana, el sector agrario evidenció una crisis permanente a lo largo de todo el siglo XX, la cual explotó con la Reforma Agraria. Existe un permanente descenso en el rendimiento de los cultivos, el cual se puede evidenciar en la constante caída en los precios a causa del comercio internacional (Santana, 2004). Esto es corroborado por Sepúlveda quien en su clásico estudio sobre la producción de trigo ha establecido como punto de inflexión el año 1926, punto en el cual comenzó su decadencia total, situación que se mantuvo hasta 1940 cuando se dejó de exportar y ya ni siquiera alcanzaba a cubrir el mercado interno(Sepúlveda, 1959). Esto habría provocado que en el período 1932 hasta 1958 se hubiese optado por un marcado intervencionismo estatal en función de los procesos de restructuración postcrisis de 1930. Comenzando con Alessandri (1920 1924) hasta la primera parte del gobierno de Ibáñez, iniciado en 1927, se tendió a controlar los precios de los productos agrícolas, y al mismo tiempo, 
a impulsar la producción agrícola en un estrecho vínculo entre los agricultores y el gobierno, sumándose a esto la planificación desde la CORFO y un aumento en los créditos agrarios (Millar y Fernández, 2008).

Esta situación ha intentado ser explicada en diversas ocasiones. Mayoritariamente se ha atribuido a la mala disposición de los agricultores al avance en sus técnicas y su consecuente falta de modernización. Si bien esto podría tener alguna evidencia, definitivamente no es la única explicación. Para Ballesteros (1965) esto se debió a dos circunstancias, a la desfavorable relación entre precios e insumos derivada de la Gran Depresión y por otra parte, a que las innovaciones desarrolladas en los países más desarrollados no tenían una implementación inmediata en los campos chilenos, sino que necesitaban de un proceso de adaptación, tanto de la innovación como del operario, y que este proceso era costoso y lento. A este diagnóstico, Santana (2004) agrega el problema del mercado urbano interno, el cual es presentado como "dual”, esto es, una demanda por alimentos básicos de muy bajo precio (trigo, papas, legumbres) por una parte, y al mismo tiempo, de productos destinados a la producción industrial (tabaco, remolacha, cáñamo) de mayor valor pero demanda limitada. Ambas categorías de productos agrícolas no estimulaban suficientemente las mejoras en productividad del sector agrario.

\section{Colchagua, primera mitad del siglo XX}

La Provincia de Colchagua ha tenido una trayectoria dispar en la primera mitad del siglo XX(ver Tabla $\mathrm{N}^{\circ} 1$ ). A comienzos de siglo estaba compuesta por los Departamentos de San Fernando y Caupolicán, que abarcaban veinticuatro comunas en total. Con las reformas impulsadas por Carlos Ibáñez del Campo a fines de la década de 1930, fue anexado a su jurisdicción los Departamentos de Rancagua y Cachapoal, pertenecientes a la desaparecida Provincia de O'Higgins y el Departamento de Santa Cruz, previamente bajo control de la Provincia de Curicó. Si bien esta organización del territorio duró lo mismo que el gobierno de su ejecutor, la intención de reconfigurar la Provincia siguió su rumbo y tras una nueva organización del territorio nacional, la Provincia de Colchagua pierde los Departamentos de Rancagua, Cachapoal y Caupolicán -el cual había estado vinculado tradicionalmente a Colchagua- y permanece Santa Cruz bajo ese dominio. En definitiva, quedaron dieciséis comunas bajo la administración de Colchagua.

La Provincia de Colchagua, por lo tanto, debe entenderse como un territorio dinámico cuyo eje central estuvo en el núcleo comprendido por San Fernando y sus alrededores. La Colchagua del primer cuarto de siglo contenía era una franja que iba de mar a cordillera siguiendo el valle del río Tinguiririca hasta su encuentro con el río Rapel (ver Tabla $N^{\circ} 1$ ). Había sido parte de los procesos de expansión de las áreas regadas de fines del siglo XIX y contaba con dos ramales del ferrocarril (Peumo y Alcones) que conectaban con zonas trigueras, construyéndose extensiones y nuevos ramales durante los primeros treinta años de siglo(Villalobos, 1990). 
Tabla $\mathbf{N}^{\circ} 1$

Número de propiedades, Provincia de Colchagua, 1915-1955

\begin{tabular}{|l|r|r|r|r|r|}
\hline \multirow{2}{*}{ Tamaño } & \multicolumn{5}{|c|}{ Años } \\
\cline { 2 - 7 } & $\mathbf{1 9 1 5}$ & $\mathbf{1 9 2 5}$ & \multicolumn{1}{|c|}{1930} & \multicolumn{1}{|c|}{1936} & \multicolumn{1}{|c|}{} \\
\hline De menos de 5 ha & 5.599 & 4.759 & 12.827 & 5.455 & 3.372 \\
\hline De 5 a 50 ha & 1.487 & 1.547 & 4.580 & 3.087 & 1.646 \\
\hline De 51 a 200 ha & 287 & 334 & 780 & 578 & 510 \\
\hline De 201 a 1000 ha & 149 & 237 & 374 & 269 & 236 \\
\hline De 1001 a 5000 ha & 49 & 80 & 150 & 95 & 108 \\
\hline De más de 5000 ha & 17 & 27 & 50 & 29 & 28 \\
\hline Total & 7.588 & 6.984 & 18.761 & 9.513 & 5.900 \\
\hline
\end{tabular}

Fuente: elaboración propia en base a Oficina Nacional de Estadística (1915, 1925, 1930, 1936, 1955

Para el año 1915, la Provincia contaba con poco más de 115.000 hectáreas de riego las cuales la convertían en una zona con muy buena aptitud para el desarrollo agrícola (Anuario Estadístico, 1916). Asimismo, contaba con 333.179 hectáreas de secano, en el cual se llevaban a cabo labores agrícolas apropiadas para la ausencia de un riego artificial. En ambos regímenes, se practicaba una agricultura tradicional de base orgánica que consistía en la puesta en marcha de un sistema de rotación cultural, donde ciertas porciones del territorio iban cambiando el cultivo que sostenían, o simplemente quedaban en reposo o "barbecho" a la espera de recuperar sus nutrientes. Este sistema permitía además controlar algunas plagas que aquejaban a los monocultivos permanentes (Opazo, 1939).

Para 1925 la superficie regada había aumentado casi en 30.000 hectáreas (143.469 en total), y la superficie de secano, destinadas a la agricultura, había subido a 597.243 (Anuario Estadístico, 1926). Si bien, hay que tomar con mucho cuidado estos datos entregados por los Anuarios Estadísticos, de todos modos muestra un aumento de la superficie destinada a la agricultura. Tal como se ha planteado anteriormente, se cree que este es un momento de expansión agrícola, ya sea a través de un avance de la gran propiedad o de un proceso de subdivisión. Lamentablemente para este periodo es difícil de cuantificar ya que las únicas herramientas contables hasta esta fecha son los Anuarios que publica el Estado, los cuales son bastante deficientes, sobre todo en lo que respecta a la cuantificación agrícola, ya que sus metodologías implicaban cuestionarios que debían ser aplicados por los subdelegados, quienes en ocasiones no lo hacían, o en otras, simplemente estimaban sus datos. Asimismo, hasta el año 1925 no existirá una 
entidad especializada en el tema, rol que vendrá a cumplir el recién formado Ministerio de Agricultura.

Una vez decantadas las reformas ibañistas, la Provincia de Colchagua quedó circunscrita a un nuevo escenario. La parte norte, correspondiente al Departamento de Caupolicán pasó a jurisdicción de una renovada Provincia de O’Higgins, pero anexó la parte sur del valle del Tinguiririca, la zona de Santa Cruz. Este cambio significó, entre otras cosas, el intercambio de un espacio de poca extensión como Caupolicán $(1.541$ km²) por uno mucho más extenso como Santa Cruz (5.324 km²). No obstante, los terrenos de Caupolicán tenían mejores suelos y una mayor superficie regada, ya que Santa Cruz contiene un alto porcentaje de cultivos de secano costero (Matthei, 1931).

\section{La Reforma Agraria en Colchagua}

Cuando se discutió la Reforma Agraria un elemento central fue el definir la superficie desde la cual se consideraría a una extensión como latifundio. Fue un arduo trabajo al interior de las instituciones a cargo, las que dieron como base de la conocida cifra de ochenta hectáreas de riego básico (80 HRB). En función de esa cifra se dio curso a las expropiaciones, las cuales no necesariamente calzaban con la cifra, pues existía además una tabla de equivalencias para terrenos de secano o con riego parcial (CORA, n.d.).

En el primer periodo de Reforma (1964-1970) se expropiaron 1.400 predios, que comprendieron 290.601 hectáreas de riego, lo que implica un 25\% de la superficie regada a nivel nacional, así como 3.373.952 de secano, de las cuales 368.305 son arables, lo que da un total de 3.565.961 hectáreas (ODEPLAN, 1971). En la parte final del proceso, interrumpido por el golpe militar de septiembre de 1973, la superficie total expropiada alcanzaba casi los diez millones de hectáreas (ver Tabla N²), con 6.401.315 hectáreas en el período 1970-1973 (Bengoa, 1983, p. 40)

Según el informe elaborado por el Comité Interamericano de Desarrollo Agrícola (CIDA), la distribución de la tenencia de la tierra arrojaba que un $73,3 \%$ de las familias agrícolas se ubican en el denominado "estrato bajo”, es decir productores en comunidades o familiares, así como los asalariados y medieros. Los problemas que aquejaban a este sector de la sociedad estaban circunscritos a tres causas principales: en primer lugar, los problemas relacionados con la concentración de recursos desde los grupos dominantes; los que derivaba en el segundo problema, los problemas de representación y de posibilidades de mejoramiento de las condiciones de vida -incluso sancionado por la Ley 8.811 de sindicalización-; y por último, que las unidades campesinas minifundistas, en su mayoría estaban ubicadas ecológicamente en zonas marginales. En definitiva, esto habría generado el problema de la marginalidad campesina, la base política de la Reforma Agraria y, por lo tanto, para quienes la disponibilidad de tierras para distribuir era esencial.(Gazmuri, 1970). 


\section{Tabla $\mathrm{N}^{\circ} 2$}

Expropiación de tierras (en hectáreas) con respecto al total de las tierras productivas de Chile (1965-1973)

\begin{tabular}{|c|c|c|c|c|c|}
\hline \multirow[b]{2}{*}{ Uso de la tierra } & \multicolumn{3}{|c|}{ Superficie arable } & \multirow{2}{*}{$\begin{array}{c}\text { Superficie } \\
\text { no arable }\end{array}$} & \multirow[b]{2}{*}{ Total } \\
\hline & Riego & Secano & Total & & \\
\hline Tierra productiva & 1.055 .349 & 2.277 .580 & 3.332 .929 & 24.829 .962 & 28.162 .891 \\
\hline Expropiaciones & 729.500 & 1.500 .900 & 2.230 .400 & 7.735 .500 & 9.965 .900 \\
\hline $\begin{array}{l}\text { Porcentaje expropiado } \\
\text { sobre el total uso } \\
\text { productivo de la tierra }\end{array}$ & $69,1 \%$ & $65,9 \%$ & $66,9 \%$ & $31,2 \%$ & $35,4 \%$ \\
\hline
\end{tabular}

Fuente: Bengoa (1983)

Para el caso de Colchagua la situación en la que se encuentra la provincia en los albores del proceso de Reforma plantea una concentración de la tierra similar a la del resto del país, con alrededor de un $70 \%$ de la superficie arable en manos de un 7\% de familias agrarias. Desde el punto de vista de esta investigación, un elemento central es sobre qué tierras se están redistribuyendo (ver Gráfico $\mathrm{N}^{\circ} 1$ ). Para el caso de Colchagua, la mayor cantidad de tierras estaba destinada a praderas naturales, sobre todo en sectores cordilleranos en las comunas de San Fernando (con terrenos en la Cordillera de los Andes) y comunas de secano como Marchigüe, Pumanque y La Estrella (en la Cordillera de la Costa) (ver Ilustración $\mathrm{N}^{\circ} 2$ ). La segunda unidad corresponde a "otras tierras" que responden a tierras en situación desconocida para la contabilidad oficial y que solo aparecieron en los datos levantados para llevar a cabo la Reforma Agraria.

\section{Gráfico $\mathrm{N}^{\circ} 1$ \\ Superficie según uso de la Tierra, 1965. Total Provincia de Colchagua (has.)}

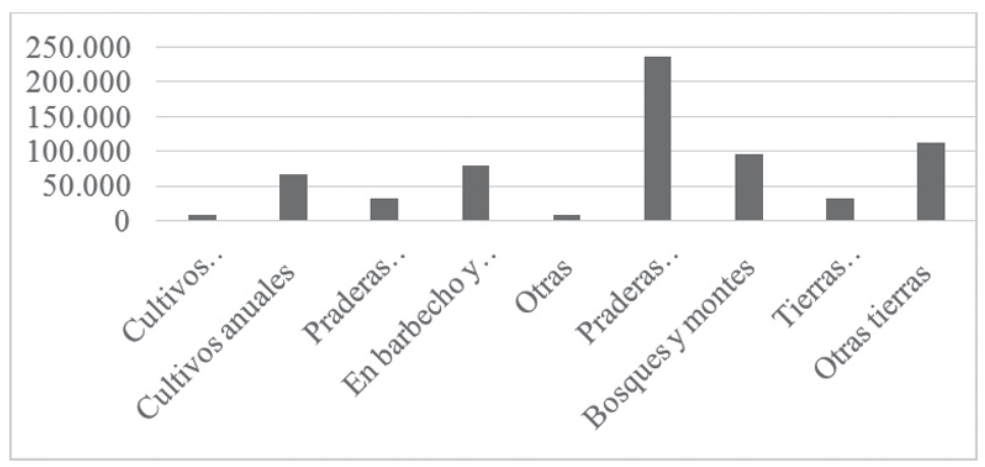

Fuente: Dirección de Estadísticas y Censos (1967) 
Si esto lo desagregamos a nivel comunal, y reagrupamos las categorías podemos ver una gran variedad de situaciones a escala local (Gráfico $\mathrm{N}^{\circ} 2$ ). En líneas generales, lo que resalta es que es un espacio altamente artificializado, pues el porcentaje de tierras potencialmente productivas no alcanza a ser un diez por ciento, salvo en dos comunas puntuales (San Fernando y Santa Cruz). Asimismo, las comunas de secano tienen una mayor presencia de praderas permanentes, las que corresponden a territorios con poca disponibilidad de riego, mientras que las que se ubican en las comunas con proximidad el curso hidrográfico principal, el río Tinguiririca, tienen mayor presencia de tierras de cultivo. Es notable el caso de San Fernando, quien posee una mayor cantidad de bosques al ser comuna con presencia en la cordillera de los Andes, no obstante poseer una cantidad importante de tierras de labranzas (17.490 hectáreas). Todo esto nos muestra lo importante que es considerar la configuración local en el análisis de los espacios reformados, pues contienen elementos de disputa por los recursos naturales, y no tan sólo la tenencia de la tierra.

\section{Gráfico $\mathrm{N}^{\circ} 2$ \\ Superficie agrícola por comuna, 1965 Colchagua}

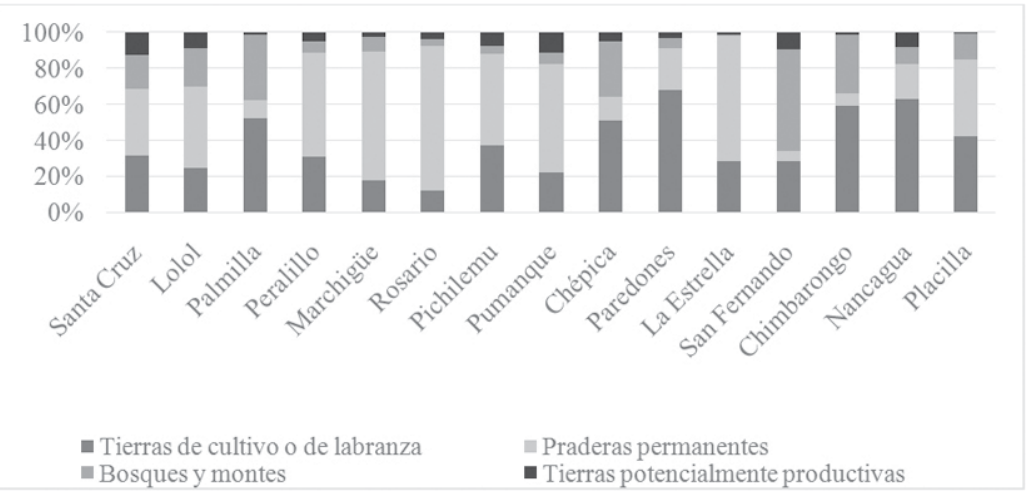

Fuente: Dirección de Estadísticas y Censos (1967)

Es en este sentido que proponemos mirar la Reforma Agraria como un tema de territorialidad. La redistribución si se mira desde un punto de vista sólo superficial, se reduce a esquemas simplificados de lo que implica una realidad agrícola. Si observamos la realidad a una escala comunal vemos que las interrelaciones presentes en los territorios presentan homogeneidades a cierta escala, como la preminencia de praderas, pero a su vez, especificidades y especializaciones productivas en otras escalas (verFigura $\mathrm{N}^{\circ} 3$ ). De este modo, mirar territorialmente la Reforma Agraria implica comprender que la redistribución no sólo estuvo concentrada en superficie, sino que en la liberación de los flujos agroecosistémicos, como 
el agua, el acceso a praderas, y a mejorar las condiciones ecológicas de las tierras disponibles para la agricultura de los asentamientos.

La provincia de Colchagua presentó un acelerado proceso de Reforma Agraria. En los primeros años logró altas tasas de expropiación que superaron la media nacional. Para el año 1971 la cantidad de distribución de tierras en sistemas colectivos ascendía ya a un 91,7\% del total de tierras en las provincias de O’Higgins y Colchagua (Collartes \& Pérez, n.d.). Desde el punto de vista de su producción, entre los años 1970-1972 aumentó la superficie destinada a cultivos anuales y a praderas artificiales, mientras que disminuyó la dedicada a cultivos industriales, aunque, si sólo se considera los suelos regados, todos los tipos muestran un aumento en la superficie dedicados a ellos. (Collartes \& Pérez, n.d.). En la tipología de los cultivos anuales lo que aumentó, en el mismo período, fueron los cereales, ya que era, por una parte, el cultivo sobre el cual se tenía mayor experiencia a nivel de cultivos, pero también porque respondía a los principios del Plan de Desarrollo Agropecuario de la Unidad Popular, que llamaba a la producción de alimentos, donde las materias primas para la industria alimenticia eran fundamentales (ODEPA, 1970).

\section{Figura $\mathrm{N}^{\circ} 3$ \\ Distribución del uso de la tierra por comunas hacia 1965}

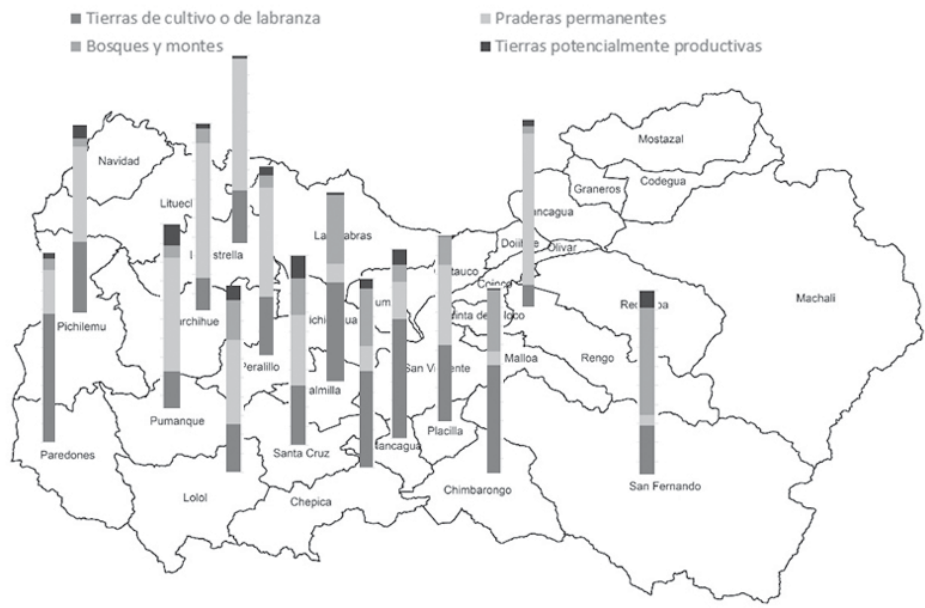

Fuente: Dirección de Estadísticas y Censos (1967) IV Censo Nacional Agropecuario: año agrícola 1964-1965. Volumen 10. Santiago, Chile.

Las expropiaciones en la provincia de Colchagua representaron la mitad del total de la superficie provincial, pero lo que resulta más significa- 
tivo, es que involucró a un 85\% de la superficie de riego de la zona, lo que implica una redistribución de las mejores tierras de la provincia, pues si bien en términos numéricos no es una parte importante del total del territorio, ésta sí responde a las tierras más productivas y con mejores condiciones agroecosistémicas (ver Tabla №3). Hacia fines de mayo de 1973 las 446.810 hectáreas estaban repartidas en 72.616,4 ha. de riego, 65.935,4 ha. de secano arable, y 308.258,6 ha. de secano no arable(Garrido, Guerrero, \& Valdés, 1988).

\section{Tabla $\mathbf{N}^{\circ} 3$}

\begin{tabular}{|l|r|l|l|l|}
\hline Colchagua & $\begin{array}{l}\text { Superficie total } \\
\text { provincial (ha) }\end{array}$ & $\begin{array}{l}\text { Superficie } \\
\text { expropiada hasta } \\
1969\end{array}$ & $\begin{array}{l}\text { Superficie } \\
\text { expropiada hasta } \\
\text { 1973 }\end{array}$ & \% del total \\
\hline Total tierra & $883.000,0$ & $60.591,0$ & $446.810,4$ & 50,6 \\
\hline $\begin{array}{l}\text { Superficie de } \\
\text { riego }\end{array}$ & $85.109,9$ & $26.010,4$ & $72.616,4$ & 85,3 \\
\hline
\end{tabular}

Fuente: Datos 1969 (Echeñique, 1970), para 1973 (ODEPA, 1979)

\section{Reflexiones finales: fin de la hacienda ¿y el latifundio?}

Cuando se da curso a la Reforma Agraria se buscaba romper con un sistema que venía operando hacía por lo menos doscientos años, generando pésimas condiciones de vida para las personas que venían desarrollando su vida al alero de dichas estructuras. A su vez, se buscaba dinamizar la actividad agropecuaria que era considerada la más atrasada de la estructura económica del país. La solución estaba en intervenir dichas estructuras sociales y productivas que sostenían un modelo de acumulación basada principalmente en la hegemonía de los recursos.

Según la lectura clásica, la solución era la restructuración del sistema de tenencia de la tierra, proceso sobre el cual se avanzaría y profundizaría a partir de la ley dictada en 1967. A partir de allí, se desarrollaría un proceso de restructuración territorial, acelerando los conflictos por el dominio y uso de los recursos. Como estrategia de profundización de la Reforma Agraria, se contemplaba el Asentamiento, como uso colectivo de la tierra, el primer proceso de aprendizaje para avanzar posteriormente hacia una parcelación individual y así, lograr las cooperativas que sostendrían el mejor funcionamiento de los sectores agrarios.

Como hemos visto en el caso analizado, se logró expropiar y distribuir una parte importante de la superficie agrícola, sobre todo la que tenía que ver con las tierras bajo sistema de riego, y una parte importante de las 
tierras de secano. Hacia mayo de 1973 había setenta y seis asentamientos, catorce cooperativas asignadas, ciento siete centros campesinos, veintidós Centros de Reforma Agraria (CERAS) y tres Centros Productivos (CEPROS), lo que da un total de doscientas veintidós organizaciones campesinas(Garrido et al., 1988). El cruce de estos dos datos nos hace suponer que existía una presencia territorial importante. No obstante, la fácil disolución, tanto por la amenaza militar como por la fragilidad de las organizaciones productivas hacen plantear la duda respecto a la consolidación real de la territorialidad de dichas organizaciones.

Si lo pensamos en perspectiva histórica, la trayectoria del campesinado al cual hacía alusión Gay en su Agricultura hasta la época previa a la Reforma Agraria, era un sector vinculado directa o indirectamente a la gran propiedad. La expropiación de la mitad de su superficie agrícola, con casi dos tercios de la superficie de riego involucrada, nos permite formarnos una imagen del impacto que esto significó en términos de dominio territorial. Otros componentes no contemplados en este estudio podrían darnos un panorama más completo, tales como las tasas de alfabetización, de participación en organizaciones sociales por parte de mujeres, escolarización, entre otras, podrían mostrar el impacto social de esa reconfiguración territorial. Esto habría significado una nueva forma de percibir el espacio rural, ya no como una mercancía ajena, sino que, como una parte fundante de nuevas relaciones entre los propios campesinos, y por lo tanto, con sus territorios. Sin embargo, es una experiencia que es interrumpida, por lo que esas nuevas configuraciones no logran asentarse de manera definitiva.

Es por esto que se cree que se trata de una Reforma Agraria inconclusa pues la ruptura del antiguo sistema de tenencia de la tierra, como base de las relaciones productivas, no se ve complementada por el control efectivo de los recursos implicados, ni con la autonomía del campesinado en la producción agrícola(Kay, 1980a), así como con el excesivo énfasis en la redistribución de tierras más que en la restructuración del sector agropecuario en su totalidad(García, 1973). Dicho de otra forma, es la relación directa con la naturaleza en su conjunto -en este caso específico, el agroecosistema- la que garantiza la emancipación del campesinado de las estructuras capitalistas de dominación, y es allí donde está la profundidad histórica del proyecto de Reforma Agraria. Por lo tanto, en este caso, no se logró superar la etapa de redistribución de tierras, hacia un control efectivo del territorio.

Por lo tanto, y puesto en perspectiva, la Reforma Agraria sólo vino a terminar con la hacienda, la cual es una forma específica de explotación de la naturaleza. Aquella construcción histórica respecto a la hacienda ha predominado en los análisis sobre este conflicto, pues es en este sistema de producción que se materializó el concepto de latifundio. Para todos los sectores, tanto los que estuvieron a favor como los que se vieron afectados, la Reforma Agraria venía a transformar para siempre la estructura productiva. No obstante, y en esto concordamos con Kay (1980b) las transfor- 
maciones en las relaciones de dominación no lograron la profundidad que aspiraba la Reforma Agraria.

La supervivencia histórica de la hacienda fue revertida a través de un proceso intenso, tanto en lo político como en lo productivo. Hoy en día vemos que las formas de explotación de la naturaleza han cambiado respecto al sistema hacendal, pero la distribución de la tierra ha seguido una trayectoria de concentración que convoca a investigar respecto a la actualidad de la Reforma Agraria en la zona de Colchagua y a qué aspectos continúan y cuáles fueron parte de una total metamorfosis. 


\section{Nota}

${ }^{1}$ Esta presentación significó el inicio de una escuela denominada "geografía cultural" que plantea la estrecha relación entre las sociedades y su entorno, con especial énfasis en el impacto del ser humano en el ambiente y las formas de vidas asociadas a él. 
Polis, Revista Latinoamericana, $N^{\circ}$ 47, 2017

\section{Bibliografía}

Altschuler, B. (2013). Territorio y desarrollo: aportes de la geografía y otras disciplinas para repensarlos. Theomai, (27-28), 64-79. Recuperado de http://revista-theomai.unq.edu.ar/numero_27-28/altschuler.pdf

Baker, A. (2003). Geography and History. Cambridge, England: Cambridge University Press.

Ballesteros, M. (1965). Desarrollo agrícola chileno, 1910-1955. Cuadernos de Economía, 2(5), 7-40.

Bauer, A. (1994). La sociedad rural chilena: desde la conquista española a nuestros días. Santiago, Chile: Editorial Andrés Bello.

Benedetti, A. (2011). Territorio: concepto integrador de la geografía contemporánea. In P. Souto (Ed.), Territorio, lugar, paisaje. Prácticas y conceptos básicos en geografía. Buenos Aires, Argentina: Editorial de la Facultad de Filosofía y Letras, Universidad de Buenos Aires.

Bengoa, J. (1983). El campesinado chileno después de la Reforma Agraria. Santiago, Chile: SUR Ediciones.

Bengoa, J. (1990). Historia Social de la Agricultura Chilena. Santiago, Chile: Ediciones SUR.

Bengoa, J. (2015). Historia rural de Chile Central. Santiago, Chile: LOM Ediciones.

Bhabha, H. (2010). DisemiNación. Tiempo, narrativa y los márgenes de la nación moderna. En H. Bhabha (Ed.), Nación y narración. Entre la ilusión de una identidad y las diferencias culturales (pp. 385-423). Buenos Aires, Argentina: Siglo XXI Editores.

Braudel, F. (1993). La identidad de Francia. Barcelona, España: Gedisa.

Campos, P., y Naredo. (1980). La energía en los sistemas agrarios. Agricultura Y Sociedad, (15), 17-113. Recuperado de http:// www.mapama.gob.es/ministerio/pags/biblioteca/revistas/pdf_ays/ a015_02.pdf

Collartes, J., y Pérez, S. (Eds.). (n.d.). El problema agrario en 100 asentamientos del valle central. Santiago, Chile: Ediciones Nueva Universidad.

Carreño, A. (1940).Monografía cultural y económica de la hacienda Santa Mariana, Rengo. (Tesis de grado) Universidad de Chile. 
CORA. (n.d.). Manual de tasaciones. Santiago, Chile: Corporación de la Reforma Agraria.

Dirección de Estadísticas y Censos (1967) IV Censo Nacional Agropecuario: año agrícola 1964-1965. Volumen 10. Santiago, Chile

Donoso, J. (1978). Casa de Campo. Barcelona, España: Seix Barral.

Echeñique, J. (1970). Las expropiaciones y la organización de asentamientos en el periodo 1965-70. In ICIRA (Ed.), Reforma Agraria Chilena: Seis ensayos de interpretación (pp. 95-110). Santiago, Chile: ICIRA.

Foster, J. B. (2004). La Ecología de Marx: Materialismo y Naturaleza. Madrid, España: El Viejo Topo.

Garavaglia, J. C. (1989). Ecosistemas y tecnología agraria: elementos para una historia social de los ecosistemas agrarios rioplatenses (17001830). Desarrollo Económico, 28(112). https://doi.org/10.2307/ 3467002

García, A. (1973). Sociología de la reforma agraria en América Latina. Buenos Aires, Argentina: Amorrortu Editores.

Garrabou, R., y Naredo, J. M. (Eds.). (2008). El paisaje en perspectiva histórica. Formación y transformación del paisaje en el mundo mediterráneo. Zaragoza, España: Prensas Universitarias de Zaragoza, Sociedad Española de Historia Agraria.

Garrido, J., Guerrero, C., y Valdés, M. S. (1988). Historia de la Reforma Agraria en Chile. Santiago, Chile: Editorial Universitaria.

Gay, C. (1862). Historia Física y Política de Chile. Agricultura. Santiago, Chile: Museo de Historia Natural.

Gazmuri, J. (1970). Asentamientos campesinos. Una evaluación de los primeros resultados de la Reforma Agraria en Chile. Buenos Aires, Argentina: DESAL. Ediciones Troquel.

Gómez, S., y Echeñique, J. (1991). La agricultura chilena. Las dos caras de la modernización. Santiago, Chile: FLACSO.

Góngora, M. (1966). Vagabundaje y sociedad fronteriza en Chile (siglos XVII a XIX). Cuadernos Del Centro de Estudios Socioeconómicos, 3(2), 1-41.

Góngora, M. (1970). Encomenderos y estancieros: estudios acerca de la constitución social aristocrática de Chile después de la conquista 1580-1660. Valparaíso: Universidad de Chile. 
Polis, Revista Latinoamericana, $N^{\circ}$ 47, 2017

Góngora, M. (1974). El origen de los inquilinos en el Valle Central. Santiago de Chile: ICIRA.

González de Molina, M., y Toledo, V. (2014). The Social Metabolism. A Socio-Ecological Theory of Historical Change. London, England: Springer.

Haesbaert, R. (2013). El mito de la desterritorialización. Del “fin de los territorios” a la multiterritorialidad. Ciudad de México, México: Siglo XXI Editores.

Jessop, B., Brenner, N., y Jones, M. (2008). Theorizing sociospatial relations. Environmental and Plannig D: Society and Space, (26), 389-401. https://doi.org/doi:10.1068/d9107

Kay, C. (1980a). El sistema señorial europeo y la hacienda latinoamericana. Ciudad de México, México: Serie Popular Era.

Kay, C. (1980b). Transformaciones de las relaciones de dominación y dependencia entre terratenientes y campesinos en Chile. Revista Mexicana de Sociología, 42(2), 751-797.

Lefebvre, H. (1978). De lo rural a lo urbano. Barcelona, España: Editorial Península.

Lefebvre, H. (2013). La producción del espacio. Madrid, España: Capitán Swing.

Ley 16.640 de Reforma Agraria. (1967).

Matthei, A. (1931). Suelos y abonos. Santiago, Chile: Imprenta Nacscimiento.

McBride, G. (1973). Chile: la tierra y su gente. Santiago, Chile: ICIRA.

Millar, R., y Fernández, J. (2008). Políticas agrarias en Chile: 1932-1958. Boletín de La Academia Chilena de La Historia, LXXIV(117), 407464.

Naredo, J. M. (2001). La modernización de la agricultura española y sus repercusiones ecológicas. In M. Gonzalez de Molina \& J. MartínezAlier (Eds.), Naturaleza Transformada (pp. 55-86). Barcelona, España: Icaria.

ODEPA. (1979). Chile: Estadísticas agropecuarias 1975-1979. Santiago, Chile: Oficina de Planificación Agrícola. Oficina de Estudios y Políticas Agrarias.

ODEPLAN. (1971). Plan de la Economía Nacional 1971-76. Antecedentes sobre el Desarrollo Chileno, 1960-70. Santiago, Chile. 
OFICINA NACIONAL DE ESTADISTICA (1915) Anuario Estadístico. SAntiago, Chile: Imprenta Nacional.

OFICINA NACIONAL DE ESTADISTICA (1925) Anuario Estadístico. SAntiago, Chile: Imprenta Nacional.

OFICINA NACIONAL DE ESTADISTICA (1930) Anuario Estadístico. SAntiago, Chile: Imprenta Nacional.

OFICINA NACIONAL DE ESTADISTICA (1936) Anuario Estadístico. SAntiago, Chile: Imprenta Nacional.

OFICINA NACIONAL DE ESTADISTICA (1955) Anuario Estadístico. SAntiago, Chile: Imprenta Nacional.

Opazo, R. (1939). Agricultura. Santiago, Chile: Talleres Gráficos La Nación S.A.

Orrego, L. (1908). Casa Grande. Santiago, Chile: Zig Zag.

Raffestin, C. (2013). Por una geografía del poder. Michoacán, México: El Colegio de Michoacán.

Ratzel, F. (1982). El territorio, la sociedad y el Estado. In J. Gómez, J. Muñoz, \& N. Ortega (Eds.), El pensamiento geográfico. Estudio interpretativo y antología de textos (pp. 193-203). Madrid, España: Alianza Editorial.

Santana, R. (2006). Agricultura Chilena en el siglo XX: contexto, actores y espacios agrícolas. Santiago, Chile: DIBAM, Centro de Investigación Diego Barros Arana.

Santos, M. (2000). La naturaleza del espacio. Técnica y tiempo. Razón y emoción. Barcelona, España: Editorial Ariel.

Sauer, C. (1940) Hacia una geografía histórica. Discurso a la Asociación Norteamericana de Geógrafos. Baton Rouge, Louisiana.

Schoennenbeck, S. (2013). Paisaje, nacion y representación del sujeto popular. Visiones de un Chile imaginado. Aisthesis, (53), 73-94. https:/ /doi.org/http://dx.doi.org/10.4067/S0718-71812013000100004

Sepúlveda, S. (1959). El trigo chileno en el mercado mundial. Santiago, Chile: Editorial Universitaria.

Urteaga, L. (1998). La historia desde una perspectiva geográfica. Lleida, España: Edicions de la Universitat de Lleida. 
Polis, Revista Latinoamericana, $N^{\circ}$ 47, 2017

Villalobos, S. (1990). Historia de la ingeniería en Chile. Santiago, Chile: Editorial Universitaria.

Recibido: 06.06.17

Aceptado: 03.07.17 